\title{
Tuberculosis and Its Predictors in Pakistani Population Transmission Dynamics and Drug Resistance of Mycobacterium
}

Fengmin Zhang ( $\sim$ fengminzhang@ems.hrbmu.edu.cn )

Harbin Medical University

\section{Sadia Jabeen}

Harbin Medical University

Yingmei Fu

Harbin Medical University

\section{Sajjad Hussain}

Harbin Medical University

\section{Sabira Tahseen}

National Tuberculosis Control Program, Pakistan

\section{Syed Mehmood Qadir}

Quaid-i-Azam University

\section{Om Prakash}

Chandka Medical College

\section{Research Article}

Keywords: Drug resistant-TB, XDR, MDR, Tuberculosis, Predictors of TB

Posted Date: March 22nd, 2021

DOl: https://doi.org/10.21203/rs.3.rs-227928/v1

License: (9) This work is licensed under a Creative Commons Attribution 4.0 International License. Read Full License 


\section{Abstract}

Drug resistance in tuberculosis (TB) is biggest growing global health concern affecting 10 million people globally with 1.2 million deaths recently. TB is a highly contagious disease predominant infecting lungs. Inappropriate health care management system poor implementation on standard remains some reasons contributing to antimicrobial resistance. Therefore, in the present context two types of tuberculosis are identified, in which the mycobacterium is resistant to isoniazid and rifampicin called as multidrug resistant tuberculosis (MDR-TB) and the other one is known as XDR-TB in which along with isoniazid and rifampin, resistance to at least one fluoroquinolone drug family and any of the second line drugs like amikacin, capreomycin, kanamycin occurs. There is a need of collecting data for frequency of occurrence of these resistant varieties for proper control and management of the disease. Thus, the current study focused on transmission dynamics and status of occurrence of drug resistance of Mycobacterium Tuberculosis in Pakistani population. The occurrence of TB bacteria in the patients of Pakistani population was tested by all three types of diagnostic methods. Smear sputum test, genetic profiling by GeneXpert test and drug susceptibility test by cultural growth on solid and liquid media was performed. The results showed that smear test of $72.9 \%$ patient were tested positive, while MTBC complex was detected in $100 \%$ patients with a variable bacterial load. The cultural growth on liquid media $83.2 \%$ while $16.8 \%$ were contaminated strains. However, in solid media $60.4 \%$ population growth was detected with $21.9 \%$ contamination. The Pakistani population was resistant against RIF is large majority while XDR and MDR resistance are not quit significant. A large number of antibiotics showed appreciable effectiveness against tuberculosis where amikacin was the most effective with Ethambutol, Pyrazinamide, Levofloxacin, Streptomycin, Kanamycin and Oxfloxacin being effective in decreasing order.

\section{Introduction}

Tuberculosis is a bacterial infection caused by Mycobacterium tuberculosis, primarily spread via inhalation of aerosolized droplets. Affecting 10 million people globally with 1.2 million deaths in the year 2018 tuberculosis (TB) is the predominant pulmonary infection that attacks the lungs; however, extrapulmonary tuberculosis may affect other parts of the body (1). TB is a highly contagious infection where only Mycobacterium tuberculosis (MTB) bacteria are enough to cause infection in a healthy individual (2). Over the past decades, several generations of antibacterial agents have been used to manage the infection with varied degree of susceptibility against the bacterium, nevertheless, ineffective regime and lack of adherence to the treatment has resulted in a higher degree of antimicrobial resistance by the pathogen. Multidrug antimicrobial resistance is more commonly reported by countries in the South-Asian region including China and Iran (3). Antimicrobial resistance is attributable to many reasons, among the causes most importantly drawbacks in health care management system remains primary. Moreover, there is no strict checking on compliance, the training of the staff remains poor, and TB control programs are inadequate. There is also a shortage of supply of drugs. In some cases, the supplied drugs are of compromised quality which cannot target the growing strong strains of bacteria with efficacy (4). Owing to all these factors discussed above TB strains are identified for two types of drug-resistant, namely; 
MDR-TB, in which resistance to at least two first line drugs of standard TB treatment (isoniazid and rifampicin) is observed, and the second being XDR-TB in which along with isoniazid and rifampin, resistance to at least one fluoroquinolone drug family and any of the second line drugs like amikacin, capreomycin, kanamycin occurs (5). According to an estimate, from the total reported Tb cases in the world, $3.6 \%$ current TB and $20.2 \%$ previously treated TB cases were multidrug-resistant TB (MDR-TB) type, with most of the rates occurring in Eastern Europe and central Asia (6). Extensive drug resistant (XDR) tuberculosis is also being reported more frequently with compromised susceptibility to both first-line and some second-line drugs including isoniazid, rifampicin, and quinolones respectively (7). Multidrugresistant tuberculosis is not only associated with higher mortality, but it is more difficult and expensive to treat (2). Acute complications are also frequent in MDR-TB where infection-related sepsis often results in life-threatening acute complications and mortality. Comorbid TB with conditions such as diabetes mellitus or confection with HIV or hepatitis $\mathrm{C}$, diabetes, and organ transplantation further reduces the quality of life of the patients due to site-specific chronic complications (8). Despite remaining one of the top 20 causes of mortality globally, MDR-TB is treatable with relevant combination of second-line and some newly introduced drugs (9). While drug-resistance is a challenge to treat, it is recommended to perform drug-susceptibility testing on the first-line drugs to identify specific drug-resistance in populations to further reduce the risk of extensive drug resistance. In addition, drug-susceptibility testing also optimizes treatment outcomes in patients receiving the second-line drugs (10). With the latest advancements in diagnostic procedure on molecular-level identification of the resistant sub-strains through expression profiling of micro RNA (miRNA) is most relevant in tailoring the treatment regime of TB patients where genetic profiling of miRNAs can serve as the most accurate potential biomarker for Mycobacterium tuberculosis, easily detectable during the early stage of infection (11). Pakistan ranks fifth among the 22 countries having high burden of MDR-TB and fourth among the countries were its treatment is a challenge for clinicians (12). Owing to inadequate availability of advanced diagnostic facilities, and timely and effective treatment of the diseases, Pakistan stands 5th among the world's highest tuberculosis (TB) reported cases and 6th among highest active drug-resistant TB cases, including MDR-TB and XDR-TB (13).

Other factors such as illiteracy, poverty, humanitarian crises and other complex conflicts further exacerbates the treatment challenges attributing to relatively higher prevalence of drug resistant tuberculosis in Pakistan than other countries $(14,15)$. Young age and previous anti-TB treatment have been identified as predictors of MDR-TB prevalence in some populations, while, marital status, being a housewife, rural residence and unemployment were also reported as risk-factors (16). The literature also highlights the need of surveillance of drug resistance among TB patients to effectively combat the global TB epidemic and prevent further antimicrobial resistance. The drug-resistant TB surveillance data has the tendency to guide the planning of TB diagnostic and treatment services, designing of appropriate treatment regimens, and monitoring the effectiveness of control and eradication interventions (17). Currently, there is a dire need to conduct large, country-level studies and/or national TB drug resistance survey in order to determine the actual burden and risk factors associated with drug resistance TB in Pakistan (18). Nevertheless, there seem to be a high burden of multidrug resistant tuberculosis in 
Pakistan yet little is known about the drug resistance patterns, prevalence, and predictors among the MDR-TB patients in Pakistan. Therefore, this research was aimed to study transmission dynamics and drug resistance of Mycobacterium Tuberculosis and its predictors in Pakistani population.

\section{Methods}

\section{Study Setting and Population}

A prospective cross-sectional study was conducted by collecting demographic, diagnostic and clinical data of 97 presumptive pulmonary tuberculosis (PTB) patients from NRL-NTP Pakistan. In addition, another 100 participants were randomly selected for a survey who were apparently healthy subjects, regarded as apparently healthy group in this study. Clinical history of previous possible TB exposure, vaccination records and demographical data including age, gender, previous Anti tuberculosis treatment and exposure history of all participants under study was also recorded. In addition, Diagnostic tests including smear test, drug susceptibility testing, and genetic testing was carried out for all 97 PTB patients.

\section{Drug Susceptibility Testing (DST)}

Drug Susceptibility testing was performed following methods. All Mycobacterium tuberculosis complex isolates were tested for first-line drug susceptibility against all first-line ant bacillary drugs namely; (isoniazid (INH), rifampicin (RIF), ethambutol (EMB), streptomycin (STP) and pyrazinamide (PZA) through the BACTEC ${ }^{T M}$ MGIT ${ }^{T M} 960$ system (Becton Dickinson Diagnostic Systems, Sparks, MD, USA). The tests were performed using the standard operating procedure according to the manufacturer's instructions. This work was performed at the NRL-National TB Program Islamabad.

\section{Isoniazid and rifampicin}

All culture isolates from patients who participated in the study were tested using phenotypic methods as per the recommendation by WHO (19). The test was performed by either the Lowenstein-Jensen proportion method or MGIT 960 (20) at the following concentrations: $0.2 \mathrm{mg} / \mathrm{mL}$ isoniazid and 40.0 $\mathrm{mg} / \mathrm{L}$ rifampicin on Löwenstein-Jensen medium and $0.1 \mathrm{mg} / \mathrm{mL}$ isoniazid and $1.0 \mathrm{mg} / \mathrm{L}$ rifampicin on MGIT 960.

\section{Fluoroquinolones.}

Testing for fluoroquinolones (moxifloxacin and levofloxacin) was also performed following standard methods, where phenotypic methods according to WHO recommendations were adopted (19). All isolates were tested for moxifloxacin at $0.5 \mathrm{mg} / \mathrm{L}$ using MGIT 960 . Isolates that were resistant to moxifloxacin were tested for resistance tolevofloxacin at $1.5 \mathrm{mg} / \mathrm{L}$ using MGIT 960 . Isolates that were susceptible to moxifloxacin at $0.5 \mathrm{mg} / \mathrm{mL}$ were assumed to be susceptible to levofloxacin at $1.5 \mathrm{mg} / \mathrm{L}$.

\section{Drug Resistance Genotyping}


Culture and DNA extraction were performed according to standard laboratory procedures as mentioned in research done by Fenner, et. al. (21). Drug resistance genotypes were identified among phenotypically drug-resistant strains by amplifying and sequencing the hot-spot regions of the genes (katG, inhA,ahpC, rpoB, and embB) that were known to confer resistance to the following drugs isoniazid, rifampin, and ethambutol.

\section{Statistical Analysis}

Mean and standard deviation of all quantitative variables including age were calculated, whereas frequency and percentage of all qualitative variables including demographic characteristics, clinical symptoms, smear results, gene analysis profiles, and culture characteristics were calculated. Pearson Chi square test was used to assess differences between the drug resistance groups and the demographic and clinical diagnosis of all PTB patients. All analyses were performed using SPSS version 21 (22).

\section{Ethics Approval}

The study was approved by the Institutional Review Board (IRB) of Ethics Committee of the CMU (TB, Malaria and HIV/AIDS), Islamabad, Pakistan. Written consent was obtained from all patients from NRLNTP who participated in the study as well as the other 100 apparently healthy individuals who participated in the survey. For all other patients, informed consent was obtained by the treating physicians/researcher.

\section{Results}

A total of 196 participants" data were collected. Out of these 196, ninety-six (96) participants were PTB patients while 100 were apparently healthy subjects. Demographic and clinical characteristics of each group were analyzed that are presented in table-1. The median age of the PTB was 30 while the participants of the apparently healthy group were mostly of 24 years of age. When the two groups were stratified by age groups, 35.4\% ( $n=34)$ were between ages $15-24,18.8 \%(n=18)$ were between $25-34$ years, and $45.8 \%(n=44)$ were in the age 35-50 years in the PTB group, while 53.5\% ( $n=53)$ were between ages $15-24,29.3 \%(n=29)$ were between $25-34$ years, and $17.2 \%(n=17)$ were in the age $35-50$ years in the apparently healthy group. In the patient group frequency of female participants $(53.1 \%, n=51)$ was more than male participants $(46.9 \%, \mathrm{n}=45)$, whereas 82 male participants $(82.8 \%)$ were from apparently healthy group in comparison to only $17(17.2 \%)$ female participants. It can be seen in the observation from table-1 that there is no patient from the apparently healthy group that is currently on treatment for tuberculosis, while 96 (97.0\%) of the 100 participants of the apparently healthy group have never had any previous anti-tuberculosis treatment histories, however, 3 patients $(3.0 \%)$ had a previous history of treatment for tuberculosis. Among the patients group, 39 individuals (40.6\%) never received any treatment for TB and 36 of them (37.5\%) were previously treated, while $21(21.9 \%)$ participants were currently receiving treatment for TB. Out of 96 participants in the study, $87(90.5 \%)$ from the patient group and $35(35.4 \%)$ from the apparently healthy group used to smoke, whereas, $9(9.5 \%)$ patient group individuals and $64(64.6 \%)$ apparently healthy group participants were non-smokers. The participants 
were also analyzed for BCG (BacilleCalmette-Guérin) vaccine in order to check their immunization against TB. It was noted that $28(29.5 \%)$ patient group participants were vaccinated by BCG vaccine and 68 (70.5\%) were not vaccinated. Among the apparently healthy group, 73 individuals (73.7\%) were BCG immunized while $26(26.3 \%)$ did not received the BCG vaccination. Both groups presented with history of contact with any TB patient in past, and it was noted that 31 (31.1\%) people from patient group came in contact with a TB patient while only $8(8.1 \%)$ individuals from apparently healthy group might have exposed to a TB patient in the past. Data for the treatment history against TB was also taken and according to table- 1 , only $10(10.5 \%)$ of the patients group participants received treatment against TB where, 85 (89.5\%) people remained un-attended with any treatment to encounter TB. The symptoms of all the presumptive TB affected people in patient groups varied. Most of the participants $(n=75,26.85)$ were recorded with problem of cough; second most recorded symptom from the data was sputum with cough $(n=65,23 \%)$, while fever, loss of appetite and weight loss was recorded in $57(20.1 \%), 48(17.0 \%), 38$ (13.4\%) participants respectively. For the detection of presence of Mycobacterium tuberculosis bacteria in the suspected patients (only PTB group), sputum smear analysis, GeneXpert analysis and cultural analysis were performed. The data of smear and gene profile is presented in table-2, while the results of cultural characteristics are listed in table-3. The sputum results were positive for 70 (72.9\%) suspected patients however, 26 individual's smear were negative. Eighty-six (89.6\%) patients were evaluated for diagnosis while 10 (10.4\%)were follow up cases GeneXpert analysis was also performed on all the 96 (100\%) participants and all 96 showed presence of Mycobacterium in their sputum, yet the bacterial load differed in all 49 tested participants. As presented in table-3, 21 (42.9\%) samples showed high bacterial load, 09 (18.4\%) medium bacterial load, while a low growth of bacteria was observed in $18(36.7 \%)$ and only $01(02 \%)$ patient's sample presented trace amounts. Resistance to rifampicin was also detected by the genotypic GeneXpert analysis, and it was found in 39 (40.6\%) participants and not detected in 57 $(59.4 \%)$ patients. Culture characteristics were analyzed by mycobacterium growth indicator tube (for liquid culture) and it was noted that 79 (83.2\%) participants showed mycobacterium growth in the media, while in $17(16.8 \%)$ individuals bacterial growth besides mycobacterium was observed as a result of any other possible contamination. The culture test on solid media was also performed on the similar individuals, in which 58(60.4\%) participants showed positive MTBC growth, culture of $21(21.9 \%)$ was contaminated with other bacteria and sputum of $17(17.7 \%)$ patients showed no growth of bacteria at all. Also, 35 (36.5\%) of the samples were Multidrug resistant (MDR), 49 (51\%) were susceptible to Isoniazid, 09 (9.4\%) of samples were susceptible to Rifampicin but resistant to one or more of the Fluoroquinolones, while only 03 (3.1\%) were resistant to Rifampicin only. Drug susceptibility testing was also performed on all samples. The results are presented in table-3. Highest susceptibility was recorded for Amikacin with $95(10.8 \%)$ samples showing effective results against mycobacterium. Ethambutol was second most effective against mycobacterium, where 87 (9.9\%) cultures were susceptible to the antibiotic. Pyrazinamide antibiotic susceptibility was found in 77 (8.8\%) cultures, Oxfloxacin to 75 (8.5 $\%$ ), while streptomycin was susceptible to $73(8.3 \%)$ samples. The Rifampicin antibiotic was successful against 58 (6.6\%) participant's cultures, 52 (5.9\%) were susceptible to Isoniazid. The Kanamycin and Capreomycin both showed susceptibility to mycobacterium in only $01(0.1 \%)$ sample, while, Oxfloxacin was not effective against any of the culture with no culture (00\%) showing susceptibility to the TB 
bacteria. Some selected samples were also tested for the effectiveness of new drugs, where it was found that Moxifloxacin effectively fought in 91 (97.8\%) out of 93 cultures tested. In 88 (94.6\%) samples from $\mathrm{n}=93$ susceptibility against Bedaquilin was recorded, Clofazimine produced successful results in $49(96.1 \%)$ of $n=51$ samples and from the 49 samples tested, 47 (95\%) produced susceptibility against Delamanid. In contrast, drug resistance was also noted, where $62(64.6 \%)$ individuals were observed resistant against Rifampicin, multi-drug resistance was present in $33(34.4 \%)$ cultures and only 01 (01.0\%) sample showed extensively drug-resistant to mycobacterium. Table 4 demonstrates correlation of drug resistance groups with demographic and clinical characteristics of all patients. A significant difference was noted between the age and drug resistance, where the highest proportion of extensive drug resistance (XDR) and multi-drug resistance (MDR) was noted among the age group 35-50 years at 0.05 level of significance. Similarly, a statistically significant difference $(p=0.002)$ was noted between previous treatment history and resistance against Rifampicin.

\section{Discussion}

In order to study the transmission dynamics of drug resistance TB, 97 patients were assessed to understand the role of demographic and clinical factors that contribute to the development of resistance against anti-tubercular drugs amongst Pakistani patients. The patients in the current study were relatively younger with almost all cases being under the age of 50 years. These findings are consistent with other studies that found tuberculosis infection most prevalent amongst young adults and those who constitute the labor force in the respective populations (23-25).

Correspondingly, in this study, it was noted about two-thirds (70\%) of the patients were MTB positive, while in only $40 \%$, rifampicin resistance was detected. These findings indicate that Rifampicin resistance amongst TB patients is prevalent but a significant proportion of the population is still susceptible to rifampicin, however, the prevalence of resistance to rifampicin is notably higher than other populations where much lower level of resistance were noted for instance in China where only $1.1 \%$ and $3.4 \%$ rifampicin resistance was detected in new and previously treated cases respectively (26). Since rifampicin is a last-line treatment modality in MDR-TB to prevent methicillin-resistant Staphylococcus aureus and Pseudomonas aeruginosa $(27,28)$, a high degree of resistance against this drug in our population is alarming and calls for more effective treatment planning and medication adherence in TB patients to reduce the burden of Rifampicin-resistant TB. The Mycobacterium growth indicator (MGIT) system was used to perform drug susceptibility testing in our study. Growth was noted in about two-third of the case, while a small number of MGIT showed contamination. Furthermore, MTBC assay is a useful and rapid technique to grow and conduct drug resistance tests including RIF and others in less than two hours (29). In the current study, the MTBC assay yielded positive results in more than 80 percent of the cases inoculated in liquid medium, while solid medium yielded MTBC growth in half of the cases only. The possible reasons for a higher contamination fraction in solid medium may be due to improper collection of sample, inappropriate transportation and or handling of the specimen during the inoculation process. 
In regards to drug resistance, four groups were identified as either susceptible or resistant to a specific class of drug. Interestingly, half $(50 \%)$ of the cases were sensitive to Isoniazid in this study, while sensitivity to RIF was noted in only 10 percent of the cases. A high proportion of Isoniazid resistant, either single-handedly or in combination, is essentially a good sign as these strains are less likely to culminate in secondary cases of TB and are associated with a significant fitness cost (24). On the other hand, RIF resistance was noted in only $3.1 \%$ cases, which is less that the global prevalence of RIF resistance outcomes (25) and other countries in the regions including China $(29.33 \%)$ and India $(5.4 \%)(30,31)$.

Susceptibility testing for other drugs yielded mixed results where Amikacin showed $99 \%$ effectiveness against MTB. Since amikacin is not easily destroyed or effected by the aminoglycoside modifying enzymes, thus its permeability into the lipopolysaccharide surface of the bacteria increases and it manages to penetrate more readily (13). Similarly, appreciable results were also obtained for Ethambutol, Pyrazinamide Levofloxacin and Streptomycin, where the effectiveness of all these drugs ranged from $87 \%-52 \%$. Despite of notable RIF and Isoniazid resistance reported by previous studies, a reasonable proportion of cases were susceptible to Rifampicin (60.4\%) and Isoniazid (54.2\%). Corroborating the results of previous studies which documented significantly low susceptibility of Kanamycin (5\%) and Oxfloxacin (24\%) (15), a very high resistance to these two drugs was noted in the present study. Moreover, the efficacy of modern drugs like Delamanid, Moxifloxacin, Bedaquilin, Clofazimine, and Linzolinid were also considerable in this study. The patients showed effectiveness in more than $90 \%$ in all of the above mentioned modern drugs precisely due to their advance mechanism of action on the bacteria whose exposure to these drugs might be limited.

In addition to drug susceptibility testing and culture analysis, the current study also assessed the role of demographic and clinical factors as well as previous treatment status in conferring drug resistance in the Pakistani population. Interestingly, younger age was found to be a significant predictor of rifampicin resistance in our population. Conversely, these findings are not in congruence with a similar study conducted in China where RIF resistance was highly prevalent among the population between the ages 41 to 60 years (32). Likewise, RIF resistance was most common in people older than 40 years of age in another study conducted in Nigeria (33). Also, MDR and XDR TB was noted more frequently among the age group 35-50 years, coinciding with the findings of Xiaocui et. al., where the occurrence of XDR and MDR was frequent in older age (32) but is in contradiction to Riffat, et. al., wherein their study, the highest proportion of MDR was noted between the age 18-45 years (34). Possible reasons underlying variation in drug resistance type and age might be life style dissimilarities between the populations. In the present study no case of resistant to RIF, MDR and XDR was prevalent in people above 50 . It can be assumed that as people of older age in Pakistan are expected to have a greater immunity than young because of the rural hard working life style a majority of the population is used to of, while the age group targeted the most is exposed to a busy under pressure lifestyle with respect to either job burdens or study load, plus they also might possess a compromised immunity due to bad eating habits. Another significant variable of the drug resistance was pretreatment. In the present study highest RIF resistance was observed in the patients that were pre-treated with TB as compared to MDR and XDR. Similar results were observed in other researches as well (35-38) which makes it clear that those patients who have been previously 
treated with RIF are more likely to develop resistance against it upon successive treatment. These trends are indicative lack of adherence, ineffective regimen, or cross contact with a drug resistant TB patient.

\section{Conclusion}

The MTB resistance against antibiotic is a growing concern and spreading around the world. The dynamics of RIF-TB, MDR-YB and XDR-TB in Pakistani population showed satisfying results, where a vast majority still showed notable effectiveness of a large group of antibiotics against tuberculosis, while few only a few were resistant. The burden of XDR-TB is insignificant while RIF resistance (64.3\%) and MDRTB (34.4\%) is a concern requiring urgent interventions to control its further propagation. The significant predictors for transmission of multi drug resistance were age group and patients with previous history of RIF treatment against TB.

\section{References}

1. Organization WH. Global tuberculosis report 2020. Geneva; 2020.

2. Mahboub B, Vats M. Tuberculosis: Current Issues in Diagnosis and Management. BoD-Books on Demand; 2013.

3. Espinal MA, Laszlo A, Simonsen L, Boulahbal F, Kim SJ, Reniero A, et al. Global trends in resistance to antituberculosis drugs. N Engl J Med. 2001;344(17):1294-303.

4. Matteelli A, Centis R, D'Ambrosio L, Sotgiu G, Tadolini M, Pontali E, et al. WHO strategies for the programmatic management of drug-resistant tuberculosis. Expert Rev Respir Med. 2016;10(9):9911002.

5. Millard J, Ugarte-Gil C, Moore DAJ. Multidrug resistant tuberculosis. Bmj. 2015;350.

6. Organization WH. Antimicrobial resistance global report on surveillance: 2014 summary. World Health Organization; 2014.

7. Shah NS, Wright A, Bai G-H, Barrera L, Boulahbal F, Martín-Casabona N, et al. Worldwide emergence of extensively drug-resistant tuberculosis. Emerg Infect Dis. 2007;13(3):380.

8. Shah M, Reed C. Complications of tuberculosis. Curr Opin Infect Dis. 2014;27(5):403-10.

9. Organization WH. The global burden of disease: 2004 update. World Health Organization; 2008.

10. Masjedi MR, Farnia P, Sorooch S, Pooramiri MV, Mansoori SD, Zarifi AZ, et al. Extensively drugresistant tuberculosis: 2 years of surveillance in Iran. Clin Infect Dis. 2006;43(7):841-7.

11. Saravanan M, Niguse S, Abdulkader M, Tsegay E, Hailekiros H, Gebrekidan A, et al. Review on emergence of drug-resistant tuberculosis (MDR \& XDR-TB) and its molecular diagnosis in Ethiopia. Microb Pathog. 2018;117:237-42.

12. Organization WH. World Health Organization Global tuberculosis repot 2013. Gevena World Heal Organ. 2013; 
13. Javed H, Bakuła Z, Pleń M, Hashmi HJ, Tahir Z, Jamil N, et al. Evaluation of genotype MTBDRplus and MTBDRsl assays for rapid detection of drug resistance in extensively drug-resistant Mycobacterium tuberculosis isolates in Pakistan. Front Microbiol. 2018;9:2265.

14. Coninx R. Tuberculosis in complex emergencies. Bull World Health Organ. 2007;85:637-40.

15. Ullah I, Javaid A, Tahir Z, Ullah O, Shah AA, Hasan F, et al. Pattern of drug resistance and risk factors associated with development of drug resistant Mycobacterium tuberculosis in Pakistan. PLoS One. 2016;11(1):e0147529.

16. Hameed S, Ahmad SR, ur Rahman MA, Nazir H, Ullah I. Drug resistance profile of Mycobacterium tuberculosis and predictors associated with the development of drug resistance. J Glob Antimicrob Resist. 2019;18:155-9.

17. Dean AS, Zignol M, Cabibbe AM, Falzon D, Glaziou P, Cirillo DM, et al. Prevalence and genetic profiles of isoniazid resistance in tuberculosis patients: A multicountry analysis of cross-sectional data. PLoS Med. 2020;17(1):e1003008.

18. Daniel O, Osman E. Prevalence and risk factors associated with drug resistant TB in South West, Nigeria. Asian Pac J Trop Med. 2011;4(2):148-51.

19. Organization WH. Updated interim critical concentrations for first-line and second-line DST. World Heal Organ Geneva, Switzerland http//www stoptb org/wg/gli/assets/documents/Updated\% 20 critical\% 20concentration\% 20table_1st\% 20and\% 202nd\% 20line\% 20drugs pdf. 2012;

20. Dickinson B. Becton Dickinson, Sparks, MD, US.

21. Fenner L, Egger M, Bodmer T, Altpeter E, Zwahlen M, Jaton K, et al. Effect of mutation and genetic background on drug resistance in Mycobacterium tuberculosis. Antimicrob Agents Chemother. 2012;56(6):3047-53.

22. IBM Corp. Released 2012. IBM SPSS Statistics for Windows. Armonk, NY: IBM Corp; 2012.

23. Oyefabi A, Adelekan B, Adetiba E, Emmanuel L, Jimoh O. Predictors of Intensive Phase Treatment Outcomes among Patients with Multi-Drug Resistant Tuberculosis in Zaria, North-Western Nigeria. J Community Med Prim Heal Care. 2020;32(2):95-107.

24. Xiao X, Wu Z-C, Chou K-C. A multi-label classifier for predicting the subcellular localization of gramnegative bacterial proteins with both single and multiple sites. PLoS One. 2011;6(6):20592.

25. Johnston JC, Shahidi NC, Sadatsafavi M, Fitzgerald JM. Treatment outcomes of multidrug-resistant tuberculosis: a systematic review and meta-analysis. PLoS One. 2009;4(9):e6914.

26. Liu Z, Zhang M, Wang J, Chen S, Wu B, Zhou L, et al. Longitudinal Analysis of Prevalence and Risk Factors of Rifampicin-Resistant Tuberculosis in Zhejiang, China. Biomed Res Int. 2020;2020.

27. Portelli S, Myung Y, Furnham N, Vedithi SC, Pires DE V, Ascher DB. Prediction of rifampicin resistance beyond the RRDR using structure-based machine learning approaches. Sci Rep. 2020;10(1):1-13.

28. Forrest GN, Tamura K. Rifampin combination therapy for nonmycobacterial infections. Clin Microbiol Rev. 2010;23(1):14-34. 
29. CDC. A New Tool to Diagnose Tuberculosis: The Xpert MTB/RIF Assay. [Internet]. Factsheets. 2020 [cited 2021 Jan 22]. Available from: https://www.cdc.gov/tb/publications/factsheets/tes

30. Shao $Y$, Yang D, Xu W, Lu W, Song H, Dai Y, et al. Epidemiology of anti-tuberculosis drug resistance in a Chinese population: current situation and challenges ahead. BMC Public Health. 2011;11(1):1-10.

31. Shivekar SS, Kaliaperumal V, Brammacharry U, Sakkaravarthy A, Raj CKV, Alagappan C, et al. Prevalence and factors associated with multidrug-resistant tuberculosis in South India. Sci Rep. 2020;10(1):1-11.

32. Wu X, Yang J, Tan G, Liu H, Liu Y, Guo Y, et al. Drug resistance characteristics of Mycobacterium tuberculosis isolates from patients with tuberculosis to twelve antituberculous drugs in China. Front Cell Infect Microbiol. 2019;9:345.

33. Ajide BA, Igbabul MM, Kanyi OI. Prevalence of Mycobacterium tuberculosis and Its Rifampicin Resistance among Patients Attending General Hospital Mararaba as a Case Study. Asian J Res Infect Dis. 2019;1-10.

34. Rifat M, Milton AH, Hall J, Oldmeadow C, Islam MA, Husain A, et al. Development of multidrug resistant tuberculosis in Bangladesh: a case-control study on risk factors. PLoS One. 2014;9(8):e105214.

35. Abdella K, Abdissa K, Kebede W, Abebe G. Drug resistance patterns of Mycobacterium tuberculosis complex and associated factors among retreatment cases around Jimma, Southwest Ethiopia. BMC Public Health. 2015;15(1):599.

36. Mulu W, Abera B, Yimer M, Hailu T, Ayele H, Abate D. Rifampicin-resistance pattern of Mycobacterium tuberculosis and associated factors among presumptive tuberculosis patients referred to Debre Markos Referral Hospital, Ethiopia: a cross-sectional study. BMC Res Notes. 2017;10(1):1-8.

37. Mulu W, Mekkonnen D, Yimer M, Admassu A, Abera B. Risk factors for multidrug resistant tuberculosis patients in Amhara National Regional State. Afr Health Sci. 2015;15(2):368-77.

38. Hirpa S, Medhin G, Girma B, Melese M, Mekonen A, Suarez P, et al. Determinants of multidrugresistant tuberculosis in patients who underwent first-line treatment in Addis Ababa: a case control study. BMC Public Health. 2013;13(1):1-9.

\section{Tables}

Table 1-Demographic and Clinical Characteristics of the Presumptive TB Patients and Apparently Healthy Individuals. 


\begin{tabular}{|c|c|c|}
\hline \multicolumn{3}{|c|}{ Demographic and Clinical Characteristics } \\
\hline & Patient Group & Apparently Healthy Group \\
\hline & $N=96$ & $\mathrm{~N}=100$ \\
\hline & frequency (\%) & frequency (\%) \\
\hline Median Age & $30.50 \pm 12.6$ & $24.00 \pm 8.42$ \\
\hline \multicolumn{3}{|l|}{ Age Groups } \\
\hline $15-24$ & $34(35.4 \%)$ & $53(53.5 \%)$ \\
\hline $25-34$ & $18(18.8 \%)$ & $29(29.3 \%)$ \\
\hline $35-50$ & $44(45.8 \%)$ & $17(17.2 \%)$ \\
\hline \multicolumn{3}{|l|}{ Gender } \\
\hline Male & $45(46.9 \%)$ & $82(82.8 \%)$ \\
\hline Female & $51(53.1)$ & $17(17.2 \%)$ \\
\hline \multicolumn{3}{|l|}{ History of previous ATT } \\
\hline Never treated & $39(40.6 \%)$ & $96(97.0 \%)$ \\
\hline Previously treated & $36(37.5 \%)$ & $03(3.0 \%)$ \\
\hline Currently on treatment & $21(21.9 \%)$ & $00(00 \%)$ \\
\hline \multicolumn{3}{|l|}{ Smoking Status } \\
\hline Smoker & $87(90.5 \%)$ & $35(35.4 \%)$ \\
\hline Non-smoker & $09(9.5 \%)$ & $64(64.6 \%)$ \\
\hline \multicolumn{3}{|l|}{ BCG Immunization } \\
\hline Yes & $28(29.5)$ & $73(73.7 \%)$ \\
\hline No & $68(70.5 \%)$ & $26(26.3 \%)$ \\
\hline \multicolumn{3}{|c|}{ History of contact with TB patient } \\
\hline Yes & $31(31.6 \%)$ & $08(8.1 \%)$ \\
\hline No & $65(68.4 \%)$ & $91(91.9 \%)$ \\
\hline \multicolumn{3}{|c|}{ Previous Treatment for TB } \\
\hline Yes & $10(10.5 \%)$ & $96(97.0 \%)$ \\
\hline No & 85 (89.5\%) & $03(3.0 \%)$ \\
\hline \multicolumn{3}{|l|}{ Clinical Symptoms } \\
\hline Cough & $75(26.8 \%)$ & \\
\hline
\end{tabular}




$\begin{array}{ll}\text { Sputum with cough } & 65(23.0 \%) \\ \text { Fever } & 57(20.1 \%) \\ \text { Loss of appetite } & 48(17.0 \%) \\ \text { Weight loss } & 38(13.4 \%)\end{array}$

Table 2 - Diagnostic Characteristics of 96 Presumptive Tuberculosis Patients

\begin{tabular}{|ll|}
\hline \multicolumn{2}{|l|}{ Smear and Gene Analysis Profile of Patients } \\
No $96(\%)$ \\
Segative \\
Positive & $26(27.1 \%)$ \\
\hline Reason for Testing & $70(72.9 \%)$ \\
Diagnosis & $86(89.6 \%)$ \\
Follow-up & $10(10.4 \%)$ \\
\hline MTB Detected & \\
Yes & $96(100 \%)$ \\
No & $00(00 \%)$ \\
Bacterial load (n=49) & $01(02 \%)$ \\
Trace & $18(36.7 \%)$ \\
Low & $09(18.4 \%)$ \\
Medium & $21(42.9 \%)$ \\
High & $39(40.6 \%)$ \\
Rifampicin Resistance & $57(59.4 \%)$ \\
Detected & \\
Not Detected & \\
\hline
\end{tabular}


Page 14/22 


\section{Culture Characteristics of Patients}

Culture Result - Liquid

Positive MTBC

79 (83.2\%)

Contaminated

$17(16.8 \%)$

Culture Result - Solid

No Growth

$17(17.7 \%)$

Positive MTBC

$58(60.4 \%)$

Contaminated

$21(21.9 \%)$

Drug Groups

MDR

$35(36.5 \%)$

Isoniazid Sensitive

$49(51.0 \%)$

Rifampicin Sensitive

$09(9.4 \%)$

Rifampicin Resistant

$03(3.1 \%)$

Drug Susceptibility

Streptomycin

$73(76.0 \%)$

Isoniazid

$52(54.2 \%)$

Rifampicin

$58(60.4 \%)$

Ethambutol

87 (90.4\%)

Pyrazinamide

$77(80.2 \%)$

Kanamycin

$01(1.0 \%)$

Amikacin

$95(99.0 \%)$

Capreomycin

$01(1.0 \%)$

Oxfloxacin

$00(00 \%)$

Levofloxacin

$75(78.1 \%)$

Susceptibility to New Drugs

Moxifloxacin ( $\mathrm{n}=93)$

91 (97.8\%)

Bedaquilin ( $n=93)$

$91(94.6 \%)$

Clofazimine $(\mathrm{n}=93)$

$88(91.7 \%)$

Linzolinid ( $\mathrm{n}=51$ )

$49(96.1 \%)$

Delamanid $(n=91)$

$89(94.8 \%)$ 


$\begin{array}{ll}\text { Drug Resistance } & \\ \text { Rifampicin } & 62(64.6 \%) \\ \text { MDR } & 33(34.4 \%) \\ \text { XDR } & 01(01.0 \%)\end{array}$

Table 3 - Drug resistance and culture profiles of 96 Presumptive Tuberculosis Patients 


\begin{tabular}{|c|c|c|c|c|}
\hline \multicolumn{5}{|c|}{ Drug Resistance by Demographic and Clinical Characteristics } \\
\hline & Rifampicin-resistant & $M D R$ & $X D R$ & $P-$ value$^{*}$ \\
\hline \multicolumn{5}{|c|}{ Demographic Characteristics } \\
\hline \multicolumn{5}{|l|}{ Age Group } \\
\hline $15-24$ & $11(32.4 \%)$ & $22(64.7 \%)$ & $01(2.9 \%)$ & \\
\hline $25-34$ & $08(44.4 \%)$ & $05(27.8 \%)$ & $05(00 \%)$ & 0.049 \\
\hline $35-50$ & $16(36.4 \%)$ & $22(50.0 \%)$ & $06(13.6 \%)$ & \\
\hline \multicolumn{5}{|l|}{ Gender } \\
\hline Male & $11(24.4 \%)$ & $27(60.0 \%)$ & $07(15.6 \%)$ & 0.070 \\
\hline Female & $24(47.1 \%)$ & $22(43.1 \%)$ & $05(9.8 \%)$ & \\
\hline \multicolumn{5}{|l|}{ BCG Immunization } \\
\hline Yes & $08(28.6 \%)$ & $18(64.3 \%)$ & $02(13.4 \%)$ & 0.265 \\
\hline No & $27(40.3 \%)$ & $31(46.3 \%)$ & $09(13.4 \%)$ & \\
\hline \multicolumn{5}{|l|}{ Previous Treatment } \\
\hline Not treated & $13(33.3 \%)$ & $22(56.4 \%)$ & $04(10.3 \%)$ & \\
\hline On Treatment & $07(19.4 \%)$ & $22(61.1 \%)$ & $07(4.2 \%)$ & 0.002 \\
\hline Previously treated & $15(71.4 \%)$ & $05(23.8 \%)$ & $01(4.8 \%)$ & \\
\hline \multicolumn{5}{|l|}{ Smoking status } \\
\hline Smoker & $04(44.4 \%)$ & $05(55.6 \%)$ & $00(00 \%)$ & 0.512 \\
\hline Non-smoker & $31(36.5 \%)$ & $44(46.3 \%)$ & $11(12.8 \%)$ & \\
\hline \multicolumn{5}{|c|}{ Clinical Characteristics } \\
\hline \multicolumn{5}{|l|}{ ESR $\otimes$} \\
\hline Normal & $04(26.7 \%)$ & $09(60.0 \%)$ & $02(13.3 \%)$ & \\
\hline Mildly Raised & $16(40.0 \%)$ & $21(52.5 \%)$ & $03(7.5 \%)$ & 0.652 \\
\hline Highly Raised & $15(36.6 \%)$ & $19(46.3 \%)$ & $07(7.5 \%)$ & \\
\hline \multicolumn{5}{|l|}{ Hemoglobin level } \\
\hline Normal & 07 (25.0\%) & $16(57.1 \%)$ & $05(17.9 \%)$ & 0.270 \\
\hline Raised & $28(41.2 \%)$ & 33 (48.5\%) & 07 (10.3\%) & \\
\hline \multicolumn{5}{|l|}{ WBC Count $\otimes$} \\
\hline Low & 01 (50.0\%) & $00(0.0 \%)$ & $01(50.0 \%)$ & \\
\hline
\end{tabular}




\begin{tabular}{|c|c|c|c|c|}
\hline Normal & $13(33.3 \%)$ & $26(66.7 \%)$ & $00(0.0 \%)$ & 0.008 \\
\hline Raised & $21(38.2 \%)$ & $23(41.8 \%)$ & $11(20.0 \%)$ & \\
\hline \multicolumn{5}{|c|}{ Neutrophil Count } \\
\hline Normal & $19(35.8 \%)$ & $28(52.8 \%)$ & $06(11.3 \%)$ & \multirow[t]{2}{*}{0.897} \\
\hline Raised & $16(37.2 \%)$ & $21(48.8 \%)$ & $06(14.0 \%)$ & \\
\hline \multicolumn{5}{|c|}{ Lymphocyte count } \\
\hline Low & $23(39.7 \%)$ & $27(46.6 \%)$ & $08(13.8 \%)$ & \multirow{3}{*}{0.822} \\
\hline Normal & $11(31.4 \%)$ & $20(57.1 \%)$ & $04(11.4 \%)$ & \\
\hline Raised & $01(33.3 \%)$ & $02(66.7 \%)$ & $00(0.0 \%)$ & \\
\hline \multicolumn{5}{|c|}{ *Pearson Chi-square test applied } \\
\hline \multicolumn{5}{|c|}{$\square$ Erythrocyte Sedimentation Rate } \\
\hline \multicolumn{5}{|c|}{ प White Blood Cell Count } \\
\hline
\end{tabular}

Table 4 - Correlation of Demographic and Clinical Characteristics of Presumptive Tuberculosis patients with the types of drug resistance. 


\begin{tabular}{|c|c|c|c|c|}
\hline \multicolumn{5}{|c|}{ Drug Resistance by Demographic and Clinical Characteristics } \\
\hline & Rifampicin-resistant & $M D R$ & $X D R$ & $P-$ value$^{*}$ \\
\hline \multicolumn{5}{|c|}{ Demographic Characteristics } \\
\hline \multicolumn{5}{|l|}{ Age Group } \\
\hline $15-24$ & $11(32.4 \%)$ & $22(64.7 \%)$ & $01(2.9 \%)$ & \\
\hline $25-34$ & $08(44.4 \%)$ & $05(27.8 \%)$ & $05(00 \%)$ & 0.049 \\
\hline $35-50$ & $16(36.4 \%)$ & $22(50.0 \%)$ & $06(13.6 \%)$ & \\
\hline \multicolumn{5}{|l|}{ Gender } \\
\hline Male & $11(24.4 \%)$ & $27(60.0 \%)$ & $07(15.6 \%)$ & 0.070 \\
\hline Female & $24(47.1 \%)$ & $22(43.1 \%)$ & $05(9.8 \%)$ & \\
\hline \multicolumn{5}{|l|}{ BCG Immunization } \\
\hline Yes & $08(28.6 \%)$ & $18(64.3 \%)$ & $02(13.4 \%)$ & 0.265 \\
\hline No & $27(40.3 \%)$ & $31(46.3 \%)$ & $09(13.4 \%)$ & \\
\hline \multicolumn{5}{|l|}{ Previous Treatment } \\
\hline Not treated & $13(33.3 \%)$ & $22(56.4 \%)$ & $04(10.3 \%)$ & \\
\hline On Treatment & $07(19.4 \%)$ & $22(61.1 \%)$ & $07(4.2 \%)$ & 0.002 \\
\hline Previously treated & $15(71.4 \%)$ & $05(23.8 \%)$ & $01(4.8 \%)$ & \\
\hline \multicolumn{5}{|l|}{ Smoking status } \\
\hline Smoker & $04(44.4 \%)$ & $05(55.6 \%)$ & $00(00 \%)$ & 0.512 \\
\hline Non-smoker & $31(36.5 \%)$ & $44(46.3 \%)$ & $11(12.8 \%)$ & \\
\hline \multicolumn{5}{|c|}{ Clinical Characteristics } \\
\hline \multicolumn{5}{|l|}{ ESR $\otimes$} \\
\hline Normal & $04(26.7 \%)$ & $09(60.0 \%)$ & $02(13.3 \%)$ & \\
\hline Mildly Raised & $16(40.0 \%)$ & $21(52.5 \%)$ & $03(7.5 \%)$ & 0.652 \\
\hline Highly Raised & $15(36.6 \%)$ & $19(46.3 \%)$ & $07(7.5 \%)$ & \\
\hline \multicolumn{5}{|l|}{ Hemoglobin level } \\
\hline Normal & 07 (25.0\%) & $16(57.1 \%)$ & $05(17.9 \%)$ & 0.270 \\
\hline Raised & $28(41.2 \%)$ & 33 (48.5\%) & 07 (10.3\%) & \\
\hline \multicolumn{5}{|l|}{ WBC Count $\otimes$} \\
\hline Low & 01 (50.0\%) & $00(0.0 \%)$ & $01(50.0 \%)$ & \\
\hline
\end{tabular}




\begin{tabular}{|lrlll} 
Normal & $13(33.3 \%)$ & $26(66.7 \%)$ & $00(0.0 \%)$ & 0.008 \\
Raised & $21(38.2 \%)$ & $23(41.8 \%)$ & $11(20.0 \%)$ & \\
Neutrophil Count & & & & \\
Normal & $19(35.8 \%)$ & $28(52.8 \%)$ & $06(11.3 \%)$ & 0.897 \\
Raised & $16(37.2 \%)$ & $21(48.8 \%)$ & $06(14.0 \%)$ & \\
Lymphocyte count & & & & \\
Low & $23(39.7 \%)$ & $27(46.6 \%)$ & $08(13.8 \%)$ & \\
Normal & $11(31.4 \%)$ & $20(57.1 \%)$ & $04(11.4 \%)$ & 0.822 \\
Raised & $01(33.3 \%)$ & $02(66.7 \%)$ & $00(0.0 \%)$ & \\
*Pearson Chi-square test applied & & & \\
$\square$ Erythrocyte Sedimentation Rate & & & \\
$\square$ White Blood Cell Count & &
\end{tabular}

\section{Figures}

\begin{tabular}{|l|l|l|}
\hline & Low & Normal \\
\hline Hemoglobin & 28 & 68 \\
\hline
\end{tabular}

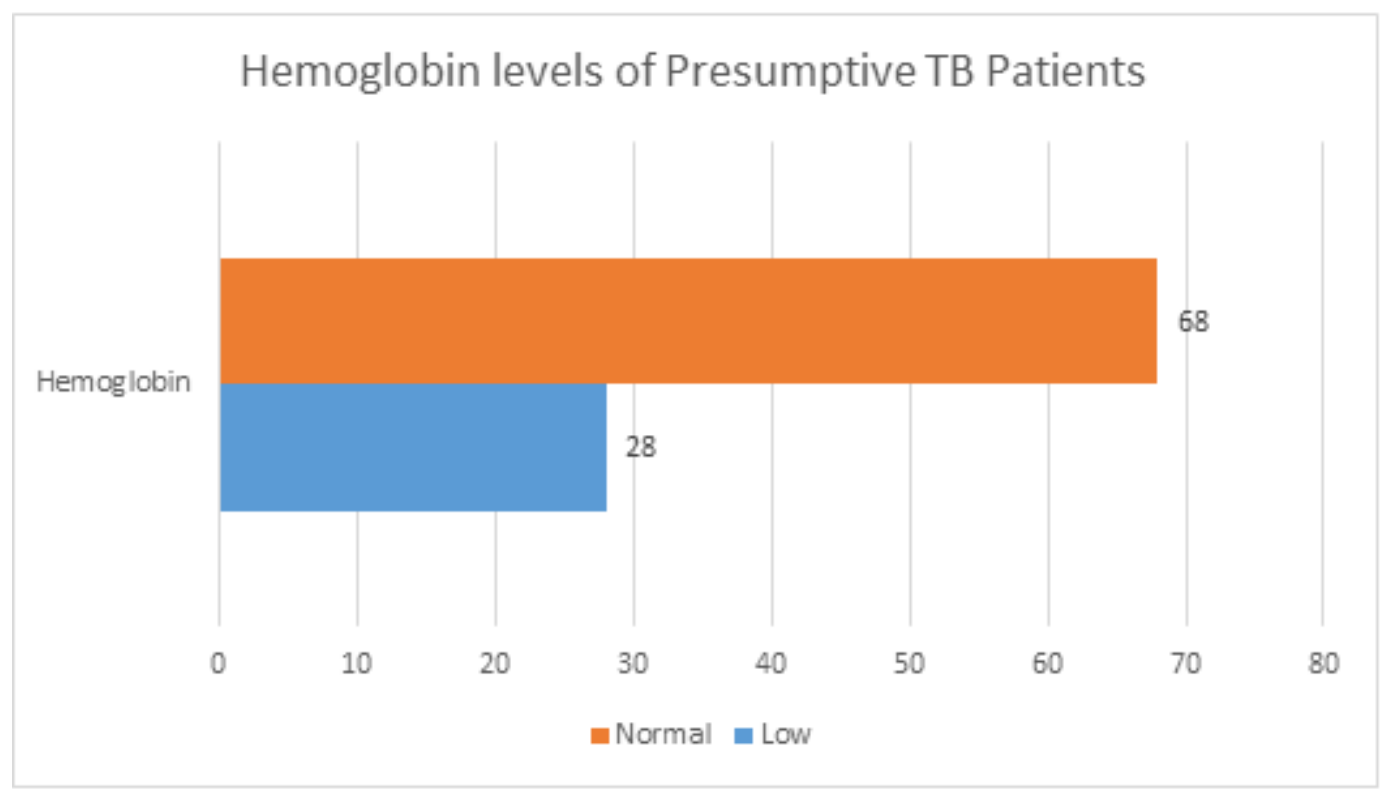

Figure 1

Clinical Characteristics of Presumptive Tuberculosis patients. 


\begin{tabular}{|ll|l|}
\hline Rifampicin & MDR & XDR \\
\hline 62 & 33 & 1 \\
\hline
\end{tabular}

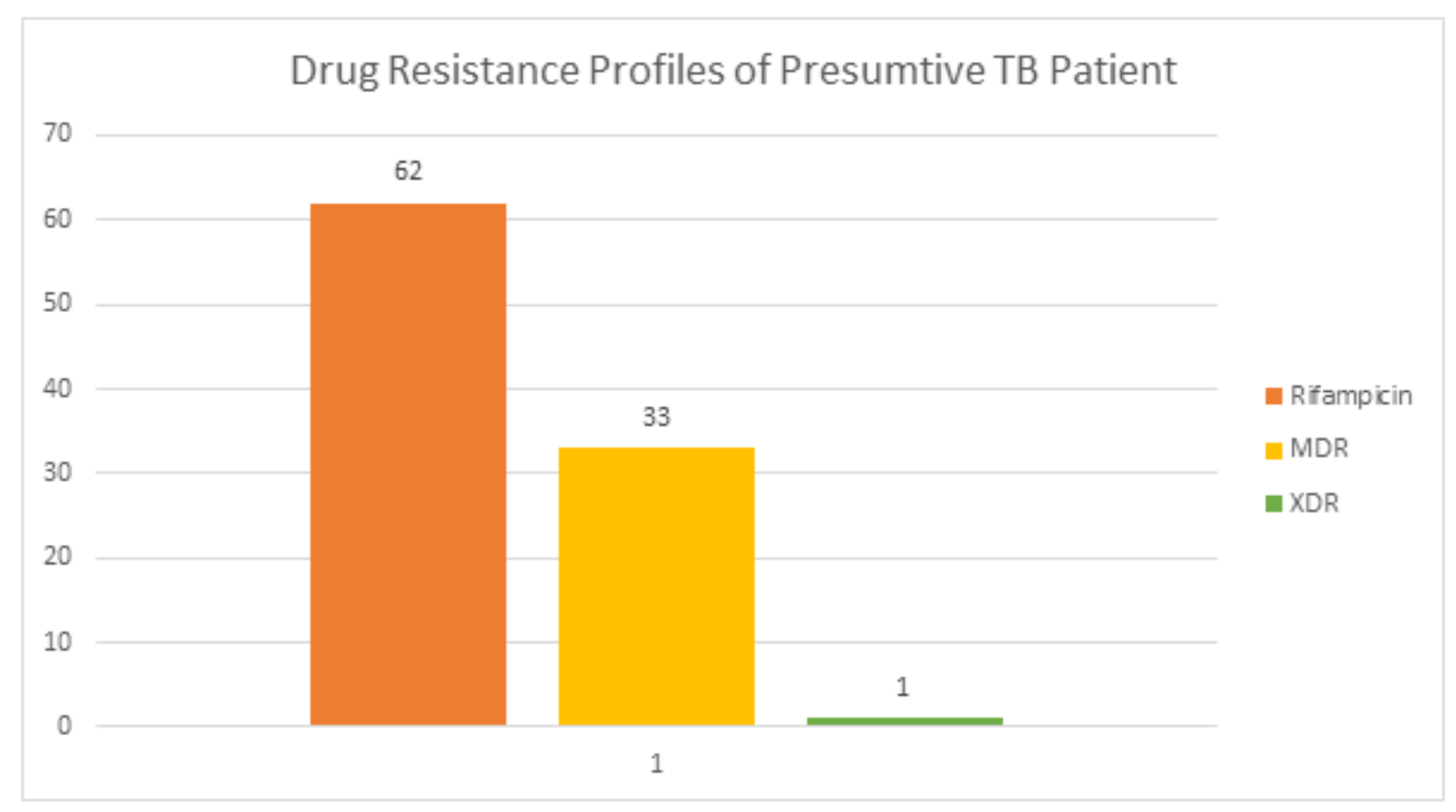

Figure 2

Demographic and Clinical Characteristics of Presumptive Tuberculosis patients with the types of drug resistance. 


\begin{tabular}{|l|l|l|l|}
\hline & $\begin{array}{l}\text { Normal } \\
15\end{array}$ & $\begin{array}{l}\text { Slightly elevated } \\
40\end{array}$ & $\begin{array}{l}\text { Highly } \\
\text { elevated } \\
41\end{array}$ \\
\hline WBCS & 2 & 39 & 55 \\
\hline $\begin{array}{l}\text { Lymphocyte } \\
\text { count } \\
\begin{array}{l}\text { Neutrophil } \\
\text { count }\end{array}\end{array}$ & 58 & 35 & 3 \\
\hline
\end{tabular}

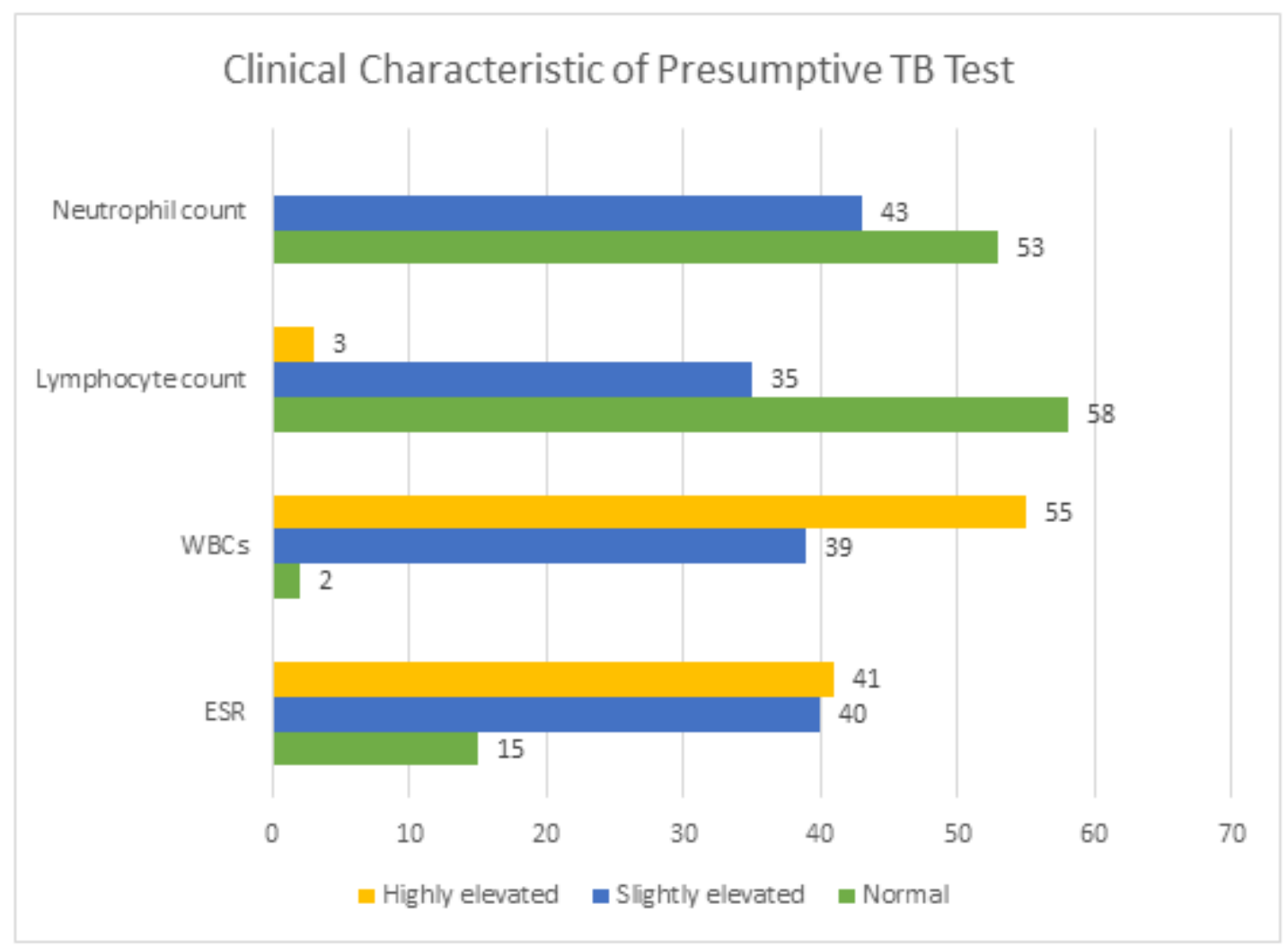

Figure 3

Clinical Characteristics of Presumptive Tuberculosis patients. 